\title{
ON THE SUMMABILITY OF THE DIFFERENTIATED FOURIER SERIES. II
}

\author{
DANIEL WATERMAN ${ }^{1}$
}

In the previous paper [1] we established the following generalization of Fatou's theorem on the Abel summability of the differentiated Fourier series.

Let $f \in L(0,2 \pi)$ of period $2 \pi$ with $\psi_{x_{0}}(t)=f\left(x_{0}+t\right)-f\left(x_{0}-t\right)$ essentially bounded ${ }^{2}$ in a neighborhood of $t=0$. Then if, for $\alpha=2, \alpha-f_{a p s}^{\prime}\left(x_{0}\right)$ $=y$, the differentiated Fourier series of $f$ is Abel summable to $y$ at $x_{0}$.

We note that $y=\alpha-f_{a p s}^{\prime}\left(x_{0}\right)$, i.e. $y$ is the $\alpha$-approximate symmetric derivative of $f$ at $x_{0}$, means that if, for any $\epsilon>0, H_{e}$ $=\left\{t:\left|y-\psi_{x_{0}}(t) / 2 t\right| \geqq \epsilon\right\}$, then $m\left(H_{\epsilon} \cap(-t, t)\right)=o\left(t^{\alpha}\right)$ as $t \rightarrow 0$.

We showed there that $\alpha=2$ cannot be replaced by a smaller value and that essentially bounded cannot be omitted.

Here we consider the question of replacing essentially bounded by a weaker condition. Clearly the differentiability condition plus the essential boundedness implies the condition

$$
\int_{0}^{t}\left|\psi_{x_{0}}(u)\right| d u=O\left(t^{2}\right)
$$

as $t \rightarrow 0$, but as we shall see, this will not replace the essential boundedness.

We will say that $f$ satisfies condition $A_{q}$ at $x_{0}$ if for some sufficiently large $M$

$$
\int_{E_{M} \cap(0, t)}\left|\psi_{x_{0}}(u)\right| d u=o\left(t^{q}\right)
$$

where $E_{M}=\left\{t:\left|\psi_{x_{0}}(t)\right| \geqq M\right\}$. Clearly $A_{q}$ implies $A_{q^{\prime}}$ for $q^{\prime}<q$.

Our result is:

The requirement of essential boundedness in the generalized Fatou theorem can be replaced by condition $A_{q}$ with $q=2$. This is best possible in the sense that $o\left(t^{2}\right)$ cannot be replaced by $O\left(t^{2}\right)$.

Throughout this paper, we suppose, as in [1], that $x_{0}=0, f(0)=0$, $y=0$, and allow $C$ to denote a positive constant not necessarily the same at each occurrence.

Received by the editors February 8, 1968.

1 This research was supported by National Science Foundation Grant GP-7358.

${ }^{2}$ In the result of [1] we assumed the essential boundedness of $f$ in a neighborhood of $x_{0}$, but this somewhat stronger result was actually proved there. 
We begin by exhibiting the counterexample required to show that our result is best possible. Choose $\beta>2$ and let $I_{k}=\left(1 / 2^{k}, 1 / 2^{k}+1 / 2^{\beta k}\right)$ and $E=\bigcup_{k=1}^{\infty} I_{k}$. Denoting the characteristic function of $I_{k}$ by $\chi_{I_{k}}$, we let

$$
f=\sum_{1}^{\infty} 2^{(\beta-2) k} \chi_{I_{k}}
$$

in $(0,2 \pi)$ and have period $2 \pi$. Then $f \in L(0,2 \pi)$ since

$$
\int_{0}^{2 \pi}|f(t)| d t=\sum_{1}^{\infty} 2^{-2 k}<\infty .
$$

For small $t, f(t)-f(-t)=0$ except for $t \in E$, and if $1 / 2^{n}<t \leqq 1 / 2^{n-1}$, then

$$
m(E \cap(0, t)) \leqq \sum_{n}^{\infty} 2^{-\beta k}=o\left(t^{2}\right) \quad \text { as } t \rightarrow 0,
$$

which shows that $\alpha-f_{\text {aps }}^{\prime}(0)=0$ for $\alpha=2$. Also

$$
t^{-2} \int_{0}^{t}|f(u)-f(-u)| d u \leqq 2^{2 n} \sum_{n-1}^{\infty} 2^{-2 k}=C<\infty .
$$

In a similar fashion we may show that condition $A_{2}$ is not satisfied. We note that $f$ satisfies $(*)$.

Let $P(r, t)$ denote the Poisson kernel. Applying the estimate

$$
-\int_{a}^{b} P_{t}(r, t) d t>C \eta r(a+b)(b-a) /\left(\eta^{4}+b^{4}\right),
$$

where $0<a<b<\pi / 2$ and $\eta=1-r$, we have, choosing $\eta=2^{-k}$,

$$
u_{t}(r, 0)>-(1 / \pi) \int_{I_{k}} 2^{(\beta-2) k} P_{t}(r, t) d t>C>0
$$

for all $k$, which establishes the negative part of our result.

We now suppose that $\psi(t)=\psi_{x_{0}}(t)$ satisfies $A_{2}$.

Write $f_{1}=f \chi_{E_{M}}, f=f_{1}+f_{2}$, and let $\psi_{1}$ and $\psi_{2}$ denote the corresponding symmetric differences. Thus $\psi=\psi_{1}+\psi_{2}$. Denoting the Abel means of the Fourier series of $f, f_{1}$, and $f_{2}$ by $u, u_{1}$, and $u_{2}$ respectively, we have $u_{t}=\left(u_{1}\right)_{t}+\left(u_{2}\right)_{t}$. Since $\psi_{2}$ is bounded and $\alpha\left(f_{2}\right)_{a p s}^{\prime}=0$ for $\alpha=2$, we have $\lim _{r \rightarrow 1}\left(u_{2}(r, 0)\right)_{t}=0$. We see then that we may assume from the onset that $f=f_{1}$.

$A_{2}$ implies that for a given $\epsilon>0$ there is a $\delta>0$ such that 


$$
\int_{0}^{t}|\psi(u)| d u<\epsilon t^{2}
$$

for $t \in(0, \delta)$. We have

$$
\left|u_{t}(r, 0)\right|<C \int_{0}^{\pi}\left|\psi(t) P_{t}(r, t)\right| d t=C\left(\int_{0}^{\delta}+\int_{0}^{\pi}\right)=g_{1}+g_{2} .
$$

Let $t_{k}=\delta 2^{-k}$ and $I_{k}=\left(t_{k+1}, t_{k}\right)$. Since $\left|P_{t}(r, t)\right|<C \eta t /\left(\eta^{4}+t^{4}\right)$, we have

$$
\begin{aligned}
\mathfrak{J}_{1} & \left.\leqq C \sum_{k=0}^{\infty} \int_{I_{k}}\left|\psi(t) P_{t}(r, t)\right| d t \leqq C \eta \sum_{k}\left(t_{k} / \eta^{4}+t_{k+1}^{4}\right)\right) \int_{I_{k}}|\psi(t)| d t \\
& <C \epsilon \eta \sum_{k} t_{k}^{3} /\left(\eta^{4}+t_{k+1}^{4}\right) .
\end{aligned}
$$

It is easily verified that

$$
2^{7} \int_{I_{k}} t^{2} /\left(\eta^{4}+t^{4}\right) d t>t_{k}^{8} /\left(\eta^{4}+t_{k+1}^{4}\right)
$$

Thus

$$
\mathfrak{g}_{1}<C \epsilon \eta \int_{0}^{\infty} t^{2} /\left(\eta^{4}+t^{4}\right) d t=C \epsilon
$$

We also have

$$
\mathscr{g}_{2} \leqq C \eta \int_{\delta}^{\pi}|\psi(t)| t /\left(\eta+t^{4}\right) d t<C \eta \delta^{-8} .
$$

Finally,

$$
\left|u_{t}(r, 0)\right|<C\left(\epsilon+\eta \delta^{-8}\right)<C \epsilon
$$

if $\eta$ is sufficiently small, the constant being independent of the choice of $\epsilon$.

\section{REFERENCES}

1. D. Waterman, On the summability of the differentiated Fourier series, Bull. Amer. Math. Soc. 73 (1967), 109-112.

WAyne State University aND

University of California, Berkeley 